Original article

\title{
Daytime sleepiness among Omani Nursing Students: Estimate of its determinants and impact on perceived stress
}

\author{
Chandrani Isac ${ }^{\mathrm{a}, *}$, Joshy Abraham ${ }^{\mathrm{b}}$ \\ ${ }^{\text {a }}$ College of Nursing, Sultan Qaboos University, P. O. Box 66, Postal Code 123, Al Khod, Muscat, Oman \\ ${ }^{\mathrm{b}}$ Armed Forces Medical Services, Ministry of Defence, School of Nursing, Oman
}

\section{A R T I C L E I N F O}

\section{Keywords:}

Daytime sleepiness

Nursing students

Academic performance

Epworth sleepiness scale

Perceived stress scale

\begin{abstract}
A B S T R A C T
Objectives: This study aimed to estimate the prevalence of daytime sleepiness among Omani Nursing Students; determine statistical linking between the personal, academic and sleep related variables of the Omani Nursing Students and their daytime sleepiness scores; and appraise the correlation between the Omani Nursing Students' daytime sleepiness scores and perceived stress scores.

Methods: A cross-sectional research design was employed. The Bachelor of Science nursing students, with an active registration status from a renowned university in Oman were included in the study. The Epworth Sleepiness Scale (ESS) and Perceived Stress Scale (PSS) were used to assess daytime sleepiness and stress index, respectively. Descriptive statistics, the independent ' $t$ ' test, and ANOVA were used to summarize the data.

Results: The prevalence of daytime sleepiness was 57.4\% (ESS > 10). Severe excessive daytime sleepiness (ESS > 16) was estimated at $12 \%$. The nursing students' ESS scores were significantly associated with use of stimulants, program study track, cohort, previous semester GPA, and sleep hours on the day before examination. A weak positive correlation was found between ESS scores and PSS scores.

Conclusion: There is a high prevalence of daytime sleepiness among Omani nursing students. There is a need for awareness programs to address daytime sleepiness. Activation of universal preventive programs on sleep hygiene, and healthy sleeping habits among nursing students is to be initiated.
\end{abstract}

\section{Introduction}

Sleep is an integral part of human living and is important for optimal physical and psychosocial wellbeing. ${ }^{1}$ Daytime sleepiness refers to uncontrolled dozing off and drowsiness during the daytime, leading to an inability to remain alert and cautious while performing daily activities. ${ }^{2}$ Daytime sleepiness can cause limitations in reaction time and vigilance, cognitive functioning, subjective fatigue, logical reasoning, work tasks and driving, workload and memory recall. ${ }^{3}$ The effect of daytime sleepiness on adolescents and university students has been well studied. Lund ${ }^{4}$ reported that over $60 \%$ of college students were classified as 'poor quality sleepers'. A review on the influence of sleep on academic performance concluded that decreased sleep of students at night has a serious impact on academic performance. ${ }^{5} \mathrm{~A}$ comprehensive search of research databases revealed that studies about sleep among students in the Middle East region are very limited. A study done by Sweileh ${ }^{6}$ among Palestinian students acknowledged the presence of sleep problems, but could not establish empirical significance between sleep quality and academic achievement. Kabrita ${ }^{7}$ conducted a study which found poor sleep quality among Lebanese university students, and this had an effect on their academic performance and health.

Sleep studies focusing on students from health sciences are very few. Medeiros ${ }^{8}$ has empirically concluded that irregularity of the sleep wake cycle and sleep deprivation influenced the learning of Brazilian medical students. Abdulghani ${ }^{9}$ reports an increased prevalence of sleep disorder among the medical students surveyed in Saudi Arabia. Huang's ${ }^{10}$ study among nursing students showed that $33 \%$ of the first-year nursing students surveyed had daytime sleepiness. An in depth analysis of sleep deprivation among nursing students in Saudi Arabia also revealed that insufficient sleep duration lowers university grades. ${ }^{11}$

\section{Background}

Sleep deprivation and sleep fragmentation contributes vastly to daytime sleepiness of university students. ${ }^{12}$ Sleep disturbance among novice nursing students, has been linked with stress. ${ }^{13}$ Sleep deprivation is prevalent among nursing students who work at night because of

\footnotetext{
* Corresponding author.

E-mail address: chandu@squ.edu.om (C. Isac).
} 
the additional wake hours required for studying. ${ }^{11}$ Sleep deficit of graduate nursing students is accentuated on the eve of their mid-term and final examination, reducing their alertness to below optimal levels. $^{10}$

Daytime sleepiness has been attributed to mobile phone usage. University students with higher smart phone use demonstrated greater daytime dysfunction, nighttime phone awakenings and poor sleep quality scores. ${ }^{14,15}$ Caffeine has been cited as a factor influencing daytime sleepiness and poor sleep quality among university students. ${ }^{16,17}$

Sleep deficit in any form has been reported to lead to significant untoward effects on the young adult population. The lifestyle and environmental changes imposed on university students, predisposes them to experience a 'sub-health' status, which comprises of a cohort of physical [weakness, muscle soreness, and dizziness] and psychological symptoms [worry, dispiritedness, and anxiety] in the absence of any defined illness. ${ }^{15}$ The health hazards which result from sleep debt may include fatigue $^{18}$ and melancholic symptoms. ${ }^{8}$ Among nursing students, daytime sleepiness and morning tiredness was significantly associated with musculoskeletal disease, abdominal pain and asthma. ${ }^{19}$

Variations in academic performance due to daytime sleepiness has been extensively reported among university students. Studies done among medical students relates irregular sleep-wake cycle and shorter length of sleep with worse academic performance. ${ }^{11}$ Grade Point Averages (GPAs) have been used by many researcher to establish the statistical linking between academic performance of health science students and their daytime sleepiness scores. ${ }^{9,19}$

Nursing students are the future professional nurses, and they are destined to work in different shifts. The nature of work done by professional nurses demands alertness and a vigilant attitude. Empirical ventures has established statistical associations between daytime sleepiness and sleep accidents, occupational accidents, and incorrect operation of medical equipment among hospital nurses. ${ }^{20}$ The persistence of daytime sleepiness in nursing students can lead them to make major life threatening medical errors, and compromise patient safety. Therefore daytime sleepiness is a major problem that needs to be addressed by nursing research.

The available literature reviewed in all research databases, shows that there is no study about day time sleepiness and its determinants in the Omani population or nursing students, despite its implication for patient safety and professional practice. Many of the studies which have focused on this problem have entrenched the need for further investigation of the determinants of daytime sleepiness with larger population $^{5}$ and to clarify its association with lifestyle and sociodemographic factors. ${ }^{16}$ Based on these limitations the objectives of the study were to:

- Estimate the prevalence of daytime sleepiness among Omani Nursing Students

- Determine statistical linking between the personal, academic and sleep related variables of the Omani Nursing Students and their daytime sleepiness scores

- Appraise the correlation between the Omani Nursing Students' daytime sleepiness scores and perceived stress scores

\section{Methods}

\subsection{Design and sample}

This cross-sectional study was conducted among the BSN (Bachelor of Science in Nursing) students in a government funded university in the Sultanate of Oman. The BSN program offered two study tracks, the regular track (traditional 4 year program) and the bridging track (2 year program for nurses with a diploma in nursing). Data was collected during the fall and spring semesters of the academic year 2016 and 2017. The inclusion criteria included, students with an active registration status who consented to participate in the study. The students who were absent during the scheduled data collection period, those who did not consent, and the freshly admitted cohort pursuing the foundation program were excluded. The response rate was $63 \%$.

\subsection{Data collection, methods, and tools}

\subsubsection{Questionnaire}

The questionnaire prepared by the researchers was intended to explore the determinants of daytime sleepiness. The closed-ended questions in this section were derived from previous empirical reports of factors which have a strong association with daytime sleepiness. These factors or determinants were categorized as personal, academic and sleep related information.

Epworth Sleepiness Scale (ESS): was utilized to estimate daytime sleepiness among the participants. The ESS was developed by Johns ${ }^{2}$ and revised in 1997. The revised ESS employed for data collection consists of 8 items. The participants are required to rate their chances of dozing during certain daily activities such as reading, riding as a passenger in a car for an hour, sitting quietly, and talking to someone over the past month. Each item is rated from 0 (would never doze) to 3 (high chance of dozing). The total score ranges from 0 to 24. An ESS score greater than 10 is defined as excessive daytime sleepiness. The ESS has acceptable reliability (0.82) and validity. In the current study the ESS Cronbach's $\alpha$ was found to be 0.65 .

The Perceived Stress Scale (PSS): was published in $1983^{21}$ and is one of the most widely used tool for measuring nonspecific perceived stress. The original tool (14 items), was reduced to 10 , because four of the items tended to perform poorly when evaluated using exploratory factor analysis. The items in the PSS ask about feelings and thoughts during the last month. Each item is rated on a 5-point scale ranging from never (0) to almost always (4). Positively worded items are reverse scored, and the ratings are summed. Scores around 13 are considered average stress and scores of 20 or higher are considered high stress. The 10 and 14-item self-report instruments have established reliability and validity $(r=0.85)$.

Permission from the authors were taken before utilizing the tools for data collection. The tools used for the study were not translated to Arabic, as the medium of education in the university is English.

\subsection{Data analysis}

The data was analysed using the Statistical Package of Social Sciences (SPSS 20.0) software. The normality of the data was established using Kurtosis (0.03) and Skewness $(-0.26)$ methods. Hence parametric tests were employed for the descriptive (mean, median, standard deviation) and inferential statistics (two tailed independent ' $\mathrm{t}$ ' test and ANOVA). Prevalence of daytime sleepiness was obtained from the frequency of nursing students who scored more than 10 on the Epworth Sleepiness scale.

\subsection{Ethical considerations}

The study was reviewed and approved by the Ethical and Research Committee of the College of Nursing (RC/29/03/16/CON). Thereafter, the researchers compiled the class lists, sought permission from the course coordinators, and scheduled a convenient time at the end of their lecture hours to collect the data. A consent form outlining the need for the study, associated risks, voluntary nature of participation and the confidentiality statement was provided to participants.

\section{Results}

The participants were predominantly female (77.9\%), below the age of 22 years $(62.8 \%)$, with no part-time work $(87.3 \%)$, unmarried (91.6\%), accommodated within the university (78.2\%), greater 
Table 1

Daytime Sleepiness of Nursing Students using ESS Scores.

\begin{tabular}{lll}
\hline ESS Levels/Scores & $\mathrm{N}$ & $\%$ \\
\hline Normal daytime sleepiness (0-6) & 107 & 42.6 \\
Mild excessive daytime sleepiness (7-10) & 55 & 21.9 \\
Moderate excessive daytime sleepiness (11-16) & 59 & 23.5 \\
Severe excessive daytime sleepiness ( $>16)$ & 30 & 12.0 \\
\hline
\end{tabular}

consumers of tea (47.6\%), and non-consumers of medications which causes sleepiness (96.4\%). Most of the students reported utilizing technology for less than $6 \mathrm{~h}$ per day (58.8\%). The participants academic profile revealed that the majority were in the regular track $(87.5 \%)$ and had a cumulative GPA between 2 and 3 (57.6\%).

Frequency distribution of the nursing students' sleep related information portrayed that most of them had a preference for midday nap ( $n=157,64.3 \%$ ), slept between 4 and 6 hours in a day (58\%), and had less than the usual hours of sleep on the day before examination $(83.5 \%)$

The majority of participants (57.4\%) surveyed had varied levels of excessive daytime sleepiness. An estimated $12 \%(n=30)$ of the nursing students had severe excessive daytime sleepiness (Table 1 ).

Table 2 identifies the determinants most prevalent among the 21 nursing students with Severe Excessive Daytime Sleepiness (SEDS). Nursing students with illness causing sleep disturbance (5 out of 21 , $23.8 \%$ ) topped the frequency list, followed by the ones taking medication which causes sleep disturbances ( 2 out of 9, 22.8\%). Among the academic variables examined, students belonging to cohort 2013 (16 out of $74,22 \%$ ) suffered more with SEDS. The variable which secured higher ranking among 'personal category', was the possession of parttime work (6 out of 31, 19.4\%).

Statistical testing with $t$-test and ANOVA revealed that there was a significant relationship between the nursing students' use of stimulant ( $\mathrm{p}<0.05$ ), study track ( $\mathrm{p}<0.05$ ), cohort $(\mathrm{p}<0.01)$, previous semester GPA ( $\mathrm{p}<0.05$ ), and sleep hours on the day before examination $(\mathrm{p}<0.01)$ and their ESS scores (Table 3).

There exists a weak a positive correlation $\left(r=0.13, R^{2}=0.0179\right)$ between the ESS scores and PSS scores of the nursing students surveyed in the study (Fig. 1).

\section{Discussion}

The prevalence of daytime sleepiness (ESS $>10$ ) in this study was $57.4 \%$ and this is quite high compared to previous studies. ${ }^{22,23}$ Kaur $^{16}$ reported the prevalence of daytime sleepiness at $50 \%$ for students pursuing professional courses. The estimates of daytime sleepiness among Turkish and Taiwanese nursing students were $10.5 \%$ and $36.6 \%$, respectively. ${ }^{10,24}$ In the current study, an alarmingly high percentage $(12 \%)$ of nursing students were found to have severe excessive daytime sleepiness (ESS > 16). The personal variables that were found to be

Table 2

Frequency Distribution of Determinants prevalent among Nursing Students with Severe Excessive Daytime Sleepiness (SEDS).

\begin{tabular}{lll}
\hline Rank & Determinants in Nursing Students & $\%$ \\
\hline I & With illness causing sleep disturbance & 23.8 \\
II & With medication(s) causing sleep disturbance & 22.8 \\
III & Cohort 2013 & 22 \\
IV & With part-time work & 19.4 \\
V & Male & 18.2 \\
VI & Sleeping less than 4 h per day & 15.4 \\
VII & Accommodated outside the university & 14.8 \\
VIII & Preference for midday nap & 14.6 \\
IX & Evening preference of circadian rhythm & 14.2 \\
X & Cumulative GPA between 2 and 3 & 13.7 \\
\hline
\end{tabular}

common among the 30 nursing students with severe excessive daytime sleepiness (SEDS) were the possession of illness causing sleep disturbance, consumption of medication causing sleep disturbance, parttime work, male gender, and accommodation outside the university. Literature review reports that insomnia, disturbances in sleep-wake cycles, delayed sleep onset, and occurrence of day time napping, are prevalent among medical students. ${ }^{6}$ Daytime sleepiness was predominant among medical students using hypnotic medications. ${ }^{25}$ Literature reiterates statistically significant relationship between involvement in part-time work and ESS score. ${ }^{26}$ Most studies of sleep among university and nursing students have reported higher levels of sleeplessness among female student, and this has been attributed to their inherent hormonal variations. ${ }^{27,28}$

The academic characteristics commonly seen among the students with SEDS were being a nursing student of cohort 2013, and possessing a cumulative GPA between 2 and 3. Medeiros ${ }^{8}$ concludes that academic pressure contributes to desynchronized sleep rhythms causing increased daytime sleepiness. The sleep related variables which were persistently present among the nursing students with SEDS were sleeping less than $4 \mathrm{~h}$ in a day, preference for midday nap and evening circadian type. Midday napping is sign of disrupted sleep-wake cycle. ${ }^{6}$ Increases stimulation and alertness from technological devices have been attributed to delayed circadian rhythm among college students. ${ }^{29}$

The current study found significant determinants of daytime sleepiness among nursing students. Use of stimulant was one of the factor empirically connected with daytime sleepiness. Stimulants which contain caffeine inhibits adenosine which is an endogenous biochemical sleep promoter. The consumption of coffee or tea at bedtime by university students has been well established in many empirical ventures. ${ }^{27}$ The nursing students in the regular track were statistically more inclined to daytime sleepiness. The regular BSN program have a higher credit load and their learning experiences in nursing is novice, compared to their bridging counterparts. Implications for this variation between the nursing students from these streams can be drawn from Huang, ${ }^{10}$ who reports that age differences between students differentiates them statistically.

The cohort in the graduating semester and the cohort exposed to a live clinical environment for the first time had demonstrated higher mean ESS score, directing the variable 'cohort' to be statistically associated with daytime sleepiness. Increasing clinical hours and intensified course load during the 2nd year of a nursing program has been attributed to higher daytime sleepiness. ${ }^{27}$

The nursing students who secures a semester GPA between 2 and 3 is battling between a need to accelerate course load and to prevent backsliding on grades which will increase their student years. In the current study, these students had demonstrated higher mean ESS scores, which had statistically established an association with daytime sleepiness. Alsaggaf et al. ${ }^{25}$ reported that medical students with excellent academic performance had significantly earlier rise times.

The universal fact that students sleep less during exam was also reflected in their statistical association with daytime sleepiness. The preference for memory based learning prevalent within the Omani nursing student is a factor which forces them to increase their learning time on the day before examination. These findings are also validated in a study done among nursing students in King Saud University. The author reported that $31.6 \%$ of the students surveyed attributed the cause for decreased sleep hours before examination to the fear of forgetting the knowledge they had gained. ${ }^{11}$

Profiling of the variables predisposing nursing students to SEDS, will serve as a predictive factor to introspect further with reliable and standardized testing tools. Teachers of these nursing students are to be empowered with the ability to identify such high risk students before they start to demonstrate an academic decline. With appropriate counseling and lifestyle modifications these targeted students can be prevented from the destructive effects of daytime sleepiness.

The lack of a strong positive correlation between the PSS scores and 
Table 3

The Personal, Academic and Sleep-related variables of Nursing Students and the Statistical Relationship with their ESS scores.

\begin{tabular}{|c|c|c|c|c|c|c|c|c|c|c|c|}
\hline & $\mathrm{N}$ & $\%$ & ESS Mean Score & Statistical tool & p Value & & $\mathrm{N}$ & $\%$ & ESS Mean Score & Statistical tool & $\mathrm{p}$ Value \\
\hline \multicolumn{12}{|l|}{ PERSONAL VARIABLES } \\
\hline Gender & & & & Two sample ' $t$ ' test & 0.068 & Use of stimulant & & & & One-way ANOVA & $0.02 *$ \\
\hline Male & 55 & 22.1 & $11.58 \pm 4.57$ & & & None & 17 & 6.9 & $9.47 \pm 4.37$ & & \\
\hline Female & 194 & 77.9 & $10.37 \pm 4.24$ & & & Coffee/Caffeine & 37 & 15.0 & $11.27 \pm 3.62$ & & \\
\hline$\underline{\text { Age }}$ & & & & One-way ANOVA & 0.132 & Tea & 117 & 47.6 & $10.37 \pm 4.16$ & & \\
\hline Less than 22 years & 157 & 62.8 & $11.06 \pm 4.38$ & & & Other Beverages & 9 & 3.7 & $11.67 \pm 6.23$ & & \\
\hline $22-23$ years & 75 & 30 & $10 \pm 4.44$ & & & Both coffee and tea & 62 & 25.2 & $11.68 \pm 4.13$ & & \\
\hline More than 24 years & 18 & 7.2 & $9.67 \pm 2.85$ & & & \multicolumn{4}{|c|}{ Rate of stimulant consumption } & One-way ANOVA & 0.345 \\
\hline Part-time work & & & & Two sample ' $t$ ' test & 0.675 & Low intake & 135 & 60.0 & $10.87 \pm 3.98$ & & \\
\hline$\overline{\text { Yes }}$ & 31 & 12.7 & $10.97 \pm 4.65$ & & & Moderate intake & 81 & 36.0 & $11.25 \pm 3.92$ & & \\
\hline No & 213 & 87.3 & $10.62 \pm 4.26$ & & & High intake & 9 & 4.0 & $9.22 \pm 6.36$ & & \\
\hline Marital status & & & & Two sample ' $t$ ' test & 0.853 & $\underline{\text { Technology use }}$ & & & & Two sample ' $t$ ' test & 0.188 \\
\hline Single & 229 & 91.6 & $10.66 \pm 4.4$ & & & Less than $6 \mathrm{~h}$ & 147 & 58.8 & $10.34 \pm 4.27$ & & \\
\hline Married & 21 & 8.4 & $10.48 \pm 3.56$ & & & More than $6 \mathrm{~h}$ & 103 & 41.2 & $11.08 \pm 4.39$ & & \\
\hline Accommodation & & & & Two sample ' $t$ ' test & 0.224 & Use of Medications & causin & g Sleep & iness & One-way ANOVA & 0.888 \\
\hline Within university & 194 & 78.2 & $10.46 \pm 4.42$ & & & Yes & 9 & 3.6 & $10.33 \pm 5.41$ & & \\
\hline Outside university & 54 & 21.8 & $11.28 \pm 4.06$ & & & No & 241 & 96.4 & $10.66 \pm 4.3$ & & \\
\hline \multicolumn{12}{|l|}{ ACADEMIC VARIABLES } \\
\hline Study track & & & & Two sample ' $t$ ' test & & Previous semester G & & & & One-way ANOVA & $0.015^{*}$ \\
\hline Regular & 217 & 87.5 & $11.01 \pm 4.18$ & & $0.02 *$ & $<2$ & 13 & 5.5 & $9.31 \pm 3.88$ & & \\
\hline Bridging & 31 & 12.5 & $8.48 \pm 4.49$ & & & $2-3$ & 116 & 49.2 & $11.38 \pm 3.99$ & & \\
\hline Cohort & & & & One-way ANOVA & $0.000 * *$ & $3-3.5$ & 73 & 30.9 & $10.49 \pm 3.92$ & & \\
\hline 2011 & 6 & 2.4 & $12.67 \pm 3.01$ & & & $>3.5$ & 34 & 14.4 & $8.88 \pm 5.7$ & & \\
\hline 2012 & 59 & 23.8 & $10.15 \pm 3.93$ & & & \multicolumn{4}{|c|}{ Current cumulative GPA } & One-way ANOVA & 0.335 \\
\hline 2013 & 66 & 26.6 & $12.27 \pm 4.35$ & & & $<2$ & 11 & 4.8 & $9.73 \pm 3.44$ & & \\
\hline 2014 & 65 & 26.2 & $10.51 \pm 3.93$ & & & $2-3$ & 132 & 57.6 & $10.94 \pm 4.29$ & & \\
\hline 2015 & 44 & 17.7 & $10.05 \pm 3.95$ & & & $3-3.5$ & 72 & 31.4 & $10.1 \pm 4.32$ & & \\
\hline & & & & & & $>3.5$ & 14 & 6.1 & $11.86 \pm 4.64$ & & \\
\hline \multicolumn{12}{|l|}{ SLEEP RELATED VARIABLES } \\
\hline Mid-day nap & & & & Two sample ' $t$ ' test & 0.971 & Sleep hours/day & & & & One-way ANOVA & 0.520 \\
\hline Yes & 157 & 64.3 & $10.63 \pm 4.48$ & & & More than $10 \mathrm{~h}$ & 7 & 2.8 & $8.86 \pm 7.01$ & & \\
\hline No & 87 & 35.7 & $10.61 \pm 4.1$ & & & $7-10 \mathrm{~h}$ & 85 & 34.0 & $10.61 \pm 4.28$ & & \\
\hline \multicolumn{4}{|c|}{ Sleep hours on the day before examinations } & One-way ANOVA & $0.000 * *$ & $4-6 \mathrm{~h}$ & 145 & 58.0 & $10.72 \pm 4.12$ & & \\
\hline Less than usual & 207 & 83.5 & $10.89 \pm 4.15$ & & & Less than $4 \mathrm{~h}$ & 13 & 5.2 & $11.85 \pm 4.49$ & & \\
\hline Less than needed to be alert & 35 & 14.1 & $10.54 \pm 4.01$ & & & & & & & & \\
\hline Normal & 6 & 2.4 & $2.5 \pm 4.72$ & & & & & & & & \\
\hline
\end{tabular}

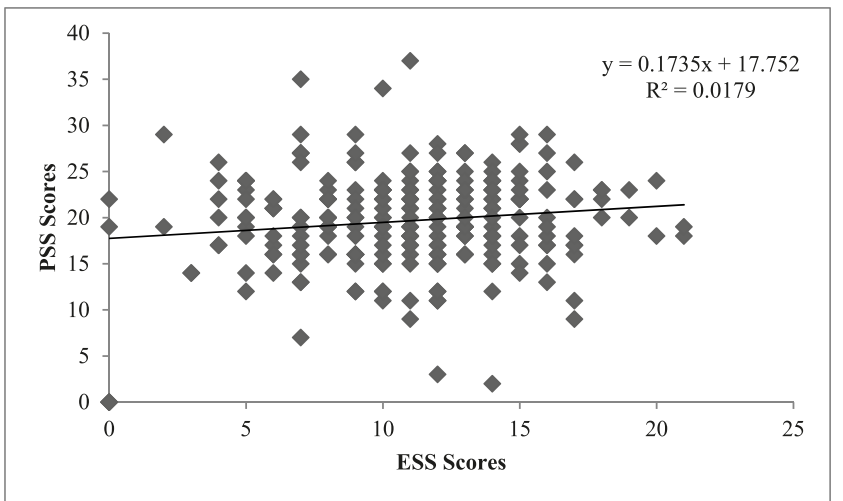

Fig. 1. Correlation between the ESS and PSS scores of the Nursing Students.

ESS scores of the nursing students is a positive stroke, amidst the higher prevalence of daytime sleepiness within this empirically tested community. Greater stress is a barrier to adopting life style changes, and may indicate psychological imbalances which requires professional counseling. Hence the group surveyed can be easily trimmed to moderate their stimulant use, alleviate fears towards their first exposure to the clinical arena, forecast and plan their academic load, and to modulate their learning styles from memory to comprehension. These findings defer the postulates of many researchers who have established a strong empirical link between stress of nursing students and their poor sleep habits. ${ }^{10,30}$

\section{Conclusion}

The high prevalence of daytime sleepiness found among the Omani nursing students directs the need for awareness programs to address this problem in this community, and to activate universal preventive programs on sleep hygiene, and healthy sleeping habits to prevent, predict and prescribe measures to eradicate it. Additionally, further studies with prospective and experimental designs are required to establish cause-effect relationships. The effects of daytime sleepiness on psychological imbalances requires additional focused explorations. The preparedness of the educators in identifying and managing the determinants of daytime sleepiness is another critical area of exploration that demands urgent attention.

\section{Limitations}

The methodological limitations of this study includes the lack of a 
random sample, use of a cross-sectional design and its inability to establish causal relationships, and the self-report method of data collection. The results have limited generalizability since the study used a sample from a single nursing institute.

\section{Funding}

Dean's fund (OMR 200).

\section{Declaration of competing interest}

The authors declare no conflicts of interest.

\section{Acknowledgement}

This research work was supported by 'Sultan Qaboos University'. The authors want to thank Dr. Joshua Muliira for meticulously editing the manuscript to a globally acceptable publication standard, and Dr. A. Mohammed Razmy for the statistical analysis and guidance. The nursing students who participated in the research are acknowledged for diligently rating their perspectives required for this research.

\section{References}

1. Haack M, Mullington JM. Sustained sleep restriction reduces emotional and physical well-being. Pain. 2005;119(1):56-64.

2. Johns MW. A new method for measuring daytime sleepiness: the Epworth sleepiness scale. Sleep. 1991;14(6):540-545.

3. Ruggiero JS, Redeker NS. Effects of napping on sleepiness and sleep-related performance deficits in night-shift workers: a systematic review. Biological research for nursing. 2014;16(2):134-142 1099800413476571.

4. Lund HG, Reider BD, Whiting AB, Prichard JR. Sleep patterns and predictors of disturbed sleep in a large population of college students. $J$ Adolesc Health. 2010;46(2):124-132.

5. Abraham J, Scaria J. Influence of Sleep in Academic Performance-An Integrated Review of Literature.

6. Sweileh WM, Ali IA, Sawalha AF, Abu-Taha AS, SeH Zyoud, Al-Jabi SW. Sleep habits and sleep problems among Palestinian students. Child Adolesc Psychiatr Ment Health. 2011;5(1):25.

7. Kabrita CS, Hajjar-Muça TA, Duffy JF. Predictors of poor sleep quality among Lebanese university students: association between evening typology, lifestyle behaviors, and sleep habits. Nat Sci Sleep. 2014;6:11.

8. Medeiros ALD, Mendes DB, Lima PF, Araujo JF. The relationships between sleepwake cycle and academic performance in medical students. Biol Rhythm Res. 2001;32(2):263-270.

9. Abdulghani HM, Alrowais NA, Bin-Saad NS, Al-Subaie NM, Haji AM, Alhaqwi AI.
Sleep disorder among medical students: relationship to their academic performance. Med Teach. 2012;34(sup1):S37-S41.

10. Huang C-F, Yang L-Y, Wu L-M, Liu Y, Chen H-M. Determinants of daytime sleepiness in first-year nursing students: a questionnaire survey. Nurse Educ Today. 2014;34(6):1048-1053.

11. Al Ghamdi AA. Sleep deprivation and academic performance of students in the collage of nursing at king Saud university. World Appl Sci J. 2013;27(2):155-167.

12. Seyedfatemi N, Tafreshi M, Hagani H. Experienced stressors and coping strategies among Iranian nursing students. BMC Nurs. 2007;6(1):1.

13. Ferreira LRC, Martino MMFD. Sleep patterns and fatigue of nursing students who work. Rev Esc Enferm USP. 2012;46(5):1178-1183.

14. Demirci K, Akgönül M, Akpinar A. Relationship of smartphone use severity with sleep quality, depression, and anxiety in university students. Journal of behavioral addictions. 2015;4(2):85-92.

15. Rosen L, Carrier LM, Miller A, Rokkum J, Ruiz A. Sleeping with technology: cognitive, affective, and technology usage predictors of sleep problems among college students. Sleep health. 2016;2(1):49-56.

16. Kaur G, Singh A. Excessive daytime sleepiness and its pattern among Indian college students. Sleep Med. 2017;29:23-28.

17. Sawah MA, Ruffin N, Rimawi M, et al. Perceived stress and coffee and energy drink consumption predict poor sleep quality in podiatric medical students: a cross-sectional study. J Am Podiatr Med Assoc. 2015;105(5):429-434.

18. Regestein Q, Natarajan V, Pavlova M, Kawasaki S, Gleason R, Koff E. Sleep debt and depression in female college students. Psychiatr Res. 2010;176(1):34-39.

19. Angelone A, Mattei A, Sbarbati M, Di Orio F. Prevalence and correlates for selfreported sleep problems among nursing students. Journal of preventive medicine and hygiene. 2011;52(4).

20. Suzuki K, Ohida T, Kaneita Y, Yokoyama E, Uchiyama M. Daytime sleepiness, sleep habits and occupational accidents among hospital nurses. J Adv Nurs. 2005;52(4):445-453

21. Cohen S, Kamarck T, Mermelstein R. A global measure of perceived stress. $J$ Health Soc Behav. 1983:385-396.

22. Kang J-H, Chen S-C. Effects of an irregular bedtime schedule on sleep quality, daytime sleepiness, and fatigue among university students in Taiwan. BMC Publ Health. 2009;9(1):248

23. Lai P-P, Say Y-H. Associated factors of sleep quality and behavior among students of two tertiary institutions in Northern Malaysia. Med J Malaysia. 2013;68(3):195-203.

24. Rosal MC, Ockene IS, Ockene JK, Barrett SV, Ma Y, Hebert JR. A longitudinal study of students' depression at one medical school. Acad Med: journal of the Association of American Medical Colleges. 1997;72(6):542-546.

25. Alsaggaf MA, Wali SO, Merdad RA, Merdad LA. Sleep quantity, quality, and insomnia symptoms of medical students during clinical years: relationship with stress and academic performance. Saudi Med J. 2016;37(2):173.

26. Silva M, Chaves C, Duarte J, Amaral O, Ferreira M. Sleep quality determinants among nursing students. Procedia: Social and Behavioral Sciences. 2016;217:999-1007.

27. Demir G. Daytime sleepiness and related factors in nursing students. Nurse Educ Today. 2017;59:21-25.

28. Roberts RE, Roberts CR, Chan W. Ethnic differences in symptoms of insomnia among adolescents. Sleep. 2006;29(3):359-365.

29. Hershner SD, Chervin RD. Causes and consequences of sleepiness among college students. Nat Sci Sleep. 2014;6:73.

30. Yamashita K, Saito M, Takao T. Stress and coping styles in Japanese nursing students. Int J Nurs Pract. 2012;18(5):489-496. 